\title{
The Picard groupoid in deformation quantization*
}

\author{
Stefan Waldmann** \\ Fakultät für Mathematik und Physik \\ Albert-Ludwigs-Universität Freiburg \\ Physikalisches Institut \\ Hermann Herder Straße 3 \\ D 79104 Freiburg \\ Germany
}

November 2003

FR-THEP 2003/22

Dedicated to Alan Weinstein for his 60th birthday

\begin{abstract}
In this letter we give an overview on recent developments in representation theory of star product algebras. In particular, we relate the ${ }^{*}$-representation theory of ${ }^{*}$-algebras over rings $\mathrm{C}=\mathrm{R}(\mathrm{i})$ with an ordered $\operatorname{ring} \mathrm{R}$ and $\mathrm{i}^{2}=-1$ to the ${ }^{*}$-representation theory of ${ }^{*}$-algebras over $\mathbb{C}$ and point out some properties of the Picard groupoid corresponding to the notion of strong Morita equivalence. Some Morita invariants are interpreted as arising from actions of this groupoid.
\end{abstract}

\section{Introduction}

The purpose of this letter is to review some recent developments in deformation quantization [4] linked to the question of finding and describing a physically useful representation theory for the star product algebras. In fact, this question will be embedded in a larger context of representation theory of associative algebras, which arises in many flavours illustrated by the following (certainly incomplete) list:

$$
\text { rings } \supseteq \underset{\text { ings with }}{\text { involutions }} \supseteq{ }_{\text {over } \mathrm{C}=\mathrm{R}(\mathrm{i})} \begin{gathered}
{ }^{*} \text {-algebras } \\
\text { over } \mathbb{C}
\end{gathered} \quad \begin{gathered}
{ }^{*} \text {-algebras } \\
\text { overentation theory of }
\end{gathered} \quad C^{*} / W^{*} \text {-algebras }
$$

Here and in the following $\mathrm{R}$ denotes an ordered ring and $\mathrm{i}^{2}=-1$. Star products belong to the middle class of algebras, whence we shall mainly focus on this type. However, it is clear that the remaining classes are of great importance in various areas of mathematics and physics as well.

One main theme of our point of view is that to each version of representation theory there is (or at least should be) an appropriate notion of Morita equivalence of the underlying algebras implying in particular that Morita equivalent algebras have equivalent categories of representations,

${ }^{*}$ Talk given at the PQR 2003 Euro-Conference in June 2003, Brussels.

**E-mail: Stefan.Waldmann@physik.uni-freiburg.de 
see e.g. $[1,17,29,34]$ and references therein for the corresponding notions of Morita equivalence. Furthermore, Morita equivalence is encoded in the existence of particular bimodules and collecting all such 'equivalence bimodules' modulo bimodule isomorphisms gives a (large) groupoid, the Picard groupoid in each of the above situations, where the multiplication is induced by tensor products of the bimodules. Moreover, the 'equivalence bimodules' implement the functorial equivalences of representation theories by tensoring with them. Thus one finally arrives at the picture that the Picard groupoid 'acts' on the representation theories. In this letter we try to make these ideas more precise, in particular for the case of *-algebras over $C=R(i)$.

Beside this algebraic framework of Morita theory there are many other notions of Morita equivalence in different areas of mathematics. Important for us is Xu's notion of Morita equivalent Poisson manifolds [37] which in some sense should be the 'classical limit' of the Morita equivalence of star product algebras. However, the precise relation is still to be explored, see in particular the discussion in $[12,18]$.

The paper is organized as follows: In Section 2 we review the motivation why one should take a look at ${ }^{*}$-representations of star product algebras. In Section 3 we discuss some general features of *-representation theory, in particular the notions of positivity arising from the ordered ring R. Here we clarify some relations to the ${ }^{*}$-representation theory of complex *-algebras. Section 4 is devoted to pre-Hilbert modules and their tensor products and gives another interpretation of the complete positivity of inner products. In Section 5 we discuss strong Morita equivalence and the resulting strong Picard groupoid while in the last section we demonstrate how some Morita invariants can be seen as arising from actions of the Picard groupoid.

Acknowledgements: It is a pleasure to thank the organizers and in particular Simone Gutt for the excellent summer school and conference. Furthermore, I would like to thank Mohamed Barakat, Henrique Bursztyn, Prof. Schmüdgen, and Alan Weinstein for valuable discussion and remarks.

\section{From star products to *-representation theory}

Let $(M, \pi)$ be a Poisson manifold. Then a formal star product $\star$ is a $\mathbb{C}[[\lambda]]$-bilinear associative product for $C^{\infty}(M)[[\lambda]]$, written as

$$
f \star g=\sum_{r=0}^{\infty} \lambda^{r} C_{r}(f, g),
$$

such that $C_{0}(f, g)=f g$ is the pointwise 'classical' product and $C_{1}(f, g)-C_{1}(g, f)=\mathrm{i}\{f, g\}$ gives the Poisson bracket induced by $\pi$. Furthermore, one requires $1 \star f=f=f \star 1$ and the $C_{r}$ are bidifferential operators [4]. If $\star$ satisfies in addition

$$
\overline{f \star g}=\bar{g} \star \bar{f},
$$

where $\lambda=\bar{\lambda}$, then $\star$ is called a Hermitian star product. Two star products $\star$ and $\star^{\prime}$ are called equivalent if there exists an operator $S=\mathrm{id}+\sum_{r=1}^{\infty} \lambda^{r} S_{r}$ where $S_{r}$ is a differential operator with $S_{r} 1=0$ such that $S(f \star g)=S f \star^{\prime} S g$. Physically interpreted, the algebra $\left(C^{\infty}(M)[[\lambda]], \star\right)$ is the algebra of observables of the quantum theory corresponding to the classical mechanical system described by $(M, \pi)$ and $\lambda$ plays the role of Planck's constant $\hbar$. The existence of star products and their classification up to equivalence is by now well-understood, both for the symplectic case $[7,20,22,30,31,36]$ and for the more general Poisson case $[19,26]$. See e.g. [21,23] for recent reviews.

In order to have a physically meaningful quantization one needs more than the algebra of observables: also the states have to be described. Since in deformation quantization the observable algebra is realized as the primary object one seeks for a derived description for the states. Similarly to and in fact motivated by algebraic quantum field theory, see e.g. [24], the positive linear 
functionals of the ${ }^{*}$-algebra $\left(C^{\infty}(M)[[\lambda]], \star,^{-}\right)$provide the physically relevant notion. Recall that a $\mathbb{C}[[\lambda]]$-linear functional $\omega: C^{\infty}(M)[[\lambda]] \longrightarrow \mathbb{C}[[\lambda]]$ is called positive if

$$
\omega(\bar{f} \star f) \geq 0
$$

in the sense of formal power series, i.e. $a \in \mathbb{R}[[\lambda]]$ with $a=\sum_{r=r_{0}}^{\infty} \lambda^{r} a_{r}$ is positive if $a_{r_{0}}>0$. Thus we enter the framework of ${ }^{*}$-algebras over $C=R(i)$ with an ordered ring $R$, in our case $R=\mathbb{R}[[\lambda]]$, as indicated in the introduction.

Having a description for the states is still not enough for a quantum mechanical theory: one also needs a way to describe superpositions of states as one of the most important physical features of quantum physics. The naive convex combination of positive linear functionals yields again a positive linear functional. However, this does not correspond to superposition but to a mixed state. Instead we need a linear structure for the states whence we have to realize states as vector states in a (pre-Hilbert) space where the observable algebra acts on by a *-representation. In general this can not be accomplished for all states simultanously whence we have to expect super-selection rules. In particular, we have to take into account all representations of the observable algebra in the beginning and find physical criteria for a selection of the 'interesting' ones afterwards. This way one is lead to the discussion of the ${ }^{*}$-representation theory of star product algebras. Of course, this well-known line of argument applies to any sort of quantum theory whose description is based on the observable algebra, like again the algebraic quantum field theory.

\section{Notions of positivity and *-representation theory}

As the order structure of $\mathbb{R}[[\lambda]]$ plays a crucial role in developing a representation theory for star products we shall now recall some notions of positivity and *-representation theory for the framework of ${ }^{*}$-algebras over $\mathrm{C}=\mathrm{R}(\mathrm{i})$ where $\mathrm{R}$ is an arbitrary ordered ring and $\mathrm{i}^{2}=-1$. The guideline for this development has been the rich theory of $C^{*}$-algebras and many results and definitions can be seen as algebraic analogs and generalizations of well-known results from $C^{*}$ algebra theory, see e.g. [27, 28,32].

In fact, as we shall deal only with algebraic features, the more appropriate analog is the theory of *-algebras over $\mathbb{C}$ and their representations by (typically) unbounded operators on complex preHilbert spaces: Representations of star product algebras usually give differential operators, even in the cases with convergence of the formal series, see e.g. [8]. Thus we shall take the opportunity to relate our results to this framework, following closely Schmüdgen's monography [35].

We start with a few basic concepts: A pre-Hilbert space over $\mathrm{C}$ is a $\mathrm{C}$-module $\mathcal{H}$ with sesquilinear inner product $\langle\cdot, \cdot\rangle: \mathcal{H} \times \mathcal{H} \longrightarrow \mathrm{C}$ such that $\langle\phi, \psi\rangle=\overline{\langle\psi, \phi\rangle}$ and $\langle\phi, \phi\rangle>0$ for $\phi \neq 0$. A map $A: \mathcal{H} \longrightarrow \mathcal{H}^{\prime}$ between pre-Hilbert spaces is called adjointable if there exists a map $A^{*}: \mathcal{H}^{\prime} \longrightarrow \mathcal{H}$ with $\langle\phi, A \psi\rangle=\left\langle A^{*} \phi, \psi\right\rangle$ for all $\psi \in \mathcal{H}$ and $\phi \in \mathcal{H}^{\prime}$. In particular, $A$ is C-linear and $A^{*}$ is unique, if it exists at all. Then $\mathfrak{B}\left(\mathcal{H}, \mathcal{H}^{\prime}\right)$ denotes the set of adjointable maps $\mathcal{H} \longrightarrow \mathcal{H}^{\prime}$. It follows that $\mathfrak{B}\left(\mathcal{H}, \mathcal{H}^{\prime}\right)$ is a C-module, the map $A \mapsto A^{*}$ induces a C-antilineare involutive bijection $\mathfrak{B}\left(\mathcal{H}, \mathcal{H}^{\prime}\right) \longrightarrow \mathfrak{B}\left(\mathcal{H}^{\prime}, \mathcal{H}\right)$ and the composition of adjointable maps is again adjointable with $(A B)^{*}=B^{*} A^{*}$. This way one obtains the category of pre-Hilbert spaces over $C$ with adjointable maps as morphisms. In particular, $\mathfrak{B}(\mathcal{H})=\mathfrak{B}(\mathcal{H}, \mathcal{H})$ is a ${ }^{*}$-algebra over $C$. Having $\mathfrak{B}(\mathcal{H})$ as 'reference' ${ }^{*}$-algebra one defines a ${ }^{*}$ representation of an arbitrary ${ }^{*}$-algebra $\mathcal{A}$ on a pre-Hilbert space $\mathcal{H}$ to be a ${ }^{*}$-homomorphism $\pi: \mathcal{A} \longrightarrow \mathfrak{B}(\mathcal{H})$. Finally, an intertwiner $T$ between two ${ }^{*}$-representations $(\mathcal{H}, \pi)$ and $\left(\mathcal{H}^{\prime}, \pi^{\prime}\right)$ is an adjointable map $T \in \mathfrak{B}\left(\mathcal{H}, \mathcal{H}^{\prime}\right)$ with $T \pi(a)=\pi^{\prime}(a) T$ for $a \in \mathcal{A}$. Two ${ }^{*}$-representations are called unitarily equivalent if there exists a unitary intertwiner. Comparing this with the case of $\mathrm{C}=\mathbb{C}$ we see that the definition of $\mathfrak{B}(\mathcal{H})$ for a complex pre-Hilbert space $\mathfrak{H}$ coincides with $\mathcal{L}^{+}(\mathcal{H})$ in [35, Prop. 2.1.8] and the definition of a ${ }^{*}$-representation is precisely the one in [35, Def. 2.1.13 and

Def. 8.1.9]. On the other hand, the intertwiners in [35, Def. 8.2.1] are required to be bounded, a 
notion which in our algebraic approach turns out to be (unfortunately) rather useless, see [9, App. A and B] for examples. However, the difference disappears for unitary equivalences as unitary maps on pre-Hilbert spaces are of course bounded. In both cases one uses the intertwiners as morphisms to define the category ${ }^{*}$-rep $(\mathcal{A})$ of ${ }^{*}$-representations of $\mathcal{A}$.

Having Morita theory in mind, the above *-representations are not yet enough to obtain a clear formulation: we rather need more general representation spaces where the inner products take their values in general *-algebras instead of $C$ only. In order to formulate the necessary positivity condition we have to specify 'positive elements' in a ${ }^{*}$-algebra. In principle, there are many possibilities: one needs to specify a $m$-admissible wedge $\mathcal{K} \subseteq \mathcal{A}$, i.e. a subset of Hermitian elements closed under convex combinations, containing the elements of the form $a^{*} a$ for $a \in \mathcal{A}$ and with $a^{*} \mathcal{K} a \subseteq \mathcal{K}$ for all $a \in \mathcal{A}$, see $[35$, p. 22] for a definition in the case of $\mathrm{C}=\mathbb{C}$, which immediately generalizes to arbitrary $\mathrm{C}$.

For a ${ }^{*}$-algebra there are two canonical m-admissible wedges. The first is given by

$$
\mathcal{A}^{++}=\left\{a \in \mathcal{A} \mid a=\sum_{i=1}^{n} \alpha_{i} a_{i}^{*} a_{i} \text { where } a_{i} \in \mathcal{A} \text { and } \mathrm{R} \ni \alpha_{i}>0\right\},
$$

also denoted by $\mathcal{P}(\mathcal{A})$ in [35], which is clearly the smallest m-admissible wedge and necessarily contained in any other. The second is described as follows: recall that a C-linear functional $\omega$ : $\mathcal{A} \longrightarrow \mathrm{C}$ is called positive if $\omega\left(a^{*} a\right) \geq 0$ for all $a \in \mathcal{A}$. Then

$$
\mathcal{A}^{+}=\{a \in \mathcal{A} \mid \omega(a) \geq 0 \text { for all positive } \omega\}
$$

is also a m-admissible wedge as $\omega_{b}(a):=\omega\left(b^{*} a b\right)$ is again a positive functional for any $b \in \mathcal{A}$. In general $\mathcal{A}^{++} \subsetneq \mathcal{A}^{+}$. Beside these two canonical wedges one can obtain other m-admissible wedges by additional choices or structures depending on which application one has in mind. One fairly general way to do this is to specify a subset of positive functionals $S$ which is closed under convex combinations and such that $\omega_{b} \in S$ for all $\omega \in S$ and $b \in \mathcal{A}$. Then

$$
\mathcal{A}_{S}^{+}=\{a \in \mathcal{A} \mid \omega(a) \geq 0 \text { for all } \omega \in S\}
$$

is again m-admissible and we have $\mathcal{A}_{S}^{+} \subseteq \mathcal{A}_{S^{\prime}}^{+}$for $S^{\prime} \subseteq S$. In particular $\mathcal{A}^{+} \subseteq \mathcal{A}_{S}^{+}$for all such $S$. If $(\mathcal{H}, \pi)$ is a ${ }^{*}$-representation then $S_{\pi}$ consisting of those positive linear functionals, which are convex combinations of vector states in $\pi$, i.e. $\omega_{\varphi}(a)=\langle\varphi, \pi(a) \varphi\rangle$ with $\varphi \in \mathcal{H}$, turns out to be such a set $S$. In case of an $O^{*}$-algebra $\mathcal{A}$, i.e. a ${ }^{*}$-subalgebra of some $\mathfrak{B}(\mathcal{H})$ this defines the positive cone of $\mathcal{A}$, using the defining representation of $\mathcal{A}$ on $\mathcal{H}$, see [35, Sect. 2.6], leading in particular to the notion of strong positivity for $O^{*}$-algebras. We note however, that only the two wedges $\mathcal{A}^{+}$and $\mathcal{A}^{++}$are canonical and the others depend on additional choices. Thus we shall stick to the choice $\mathcal{A}^{+}$for the 'positive elements' of $\mathcal{A}$ and base all our notions of positivity on $\mathcal{A}^{+}$. In particular, it seems the 'correct' choice in deformation quantization: At least for the classical limit $C^{\infty}(M)$ this definition reproduces, by Riesz representation theorem, the smooth functions on a manifold which have non-negative values, see [14, App. B].

\section{Pre-Hilbert modules and tensor products}

In order to generalize the notion of *-representations to more general representation spaces we state the following definitions [17]. Let $\mathcal{D}$ be an auxilliary ${ }^{*}$-algebra over $\mathcal{C}$ and let $\mathcal{H}_{\mathcal{D}}$ be a right $\mathcal{D}$-module. We always assume that modules have an underlying compatible C-module structure and that in the case of unital *-algebras the unit acts as identity on the module. Then a $\mathcal{D}$-valued inner product on $\mathcal{H}$ is a $\mathrm{C}$-sesquilinear map (linear in the second argument)

$$
\langle\cdot, \cdot\rangle_{\mathcal{D}}: \mathcal{H}_{\mathcal{D}} \times \mathcal{H}_{\mathcal{D}} \longrightarrow \mathcal{D}
$$


such that $\langle x, y\rangle_{\mathcal{D}}=\left(\langle y, x\rangle_{\mathcal{D}}\right)^{*}$ and $\langle x, y \cdot d\rangle=\langle x, y\rangle_{\mathcal{D}} d$ for all $x, y \in \mathcal{H}_{\mathcal{D}}$ and $d \in \mathcal{D}$. It is called non-degenerate if $\langle x, y\rangle_{\mathcal{D}}=0$ for all $y$ implies $x=0$. In this case $\left(\mathcal{H}_{\mathcal{D}},\langle\cdot, \cdot\rangle_{\mathcal{D}}\right)$ is called an inner product $\mathcal{D}$-module. Then the characterization of adjointable operators as in the case of preHilbert spaces carries over and gives C-modules $\mathfrak{B}\left(\mathcal{H}_{\mathcal{D}}, \mathcal{H}^{\prime}{ }_{\mathcal{D}}\right)$ and a ${ }^{*}$-algebra $\mathfrak{B}\left(\mathcal{H}_{\mathcal{D}}\right)$. Note that adjointable maps are always $\mathcal{D}$-linear to the right. The inner product is called completely positive if the matrix $\left(\left\langle x_{i}, x_{j}\right\rangle_{\mathcal{D}}\right) \in M_{n}(\mathcal{D})^{+}$is positive for all $n \in \mathbb{N}$ and $x_{1}, \ldots, x_{n} \in \mathcal{D}$. If $\langle\cdot, \cdot\rangle_{\mathcal{D}}$ is non-degenerate and completely positive then $\left(\mathcal{H}_{\mathcal{D}},\langle\cdot, \cdot\rangle_{\mathcal{D}}\right)$ is called a pre-Hilbert $\mathcal{D}$-module. Then a ${ }^{*}$-representation $\pi$ of a ${ }^{*}$-algebra $\mathcal{A}$ on a pre-Hilbert $\mathcal{D}$-module $\mathcal{H}_{\mathcal{D}}$ is a ${ }^{*}$-homomorphism $\pi: \mathcal{A} \longrightarrow$ $\mathfrak{B}\left(\mathcal{H}_{\mathcal{D}}\right)$. In particular, $\mathcal{H}_{\mathcal{D}}$ becomes a $(\mathcal{A}, \mathcal{D})$-bimodule. Clearly, the notion of intertwiners carries over to this framework whence we obtain the category ${ }^{*}-\operatorname{rep}_{\mathcal{D}}(\mathcal{A})$. If we drop the positivity condition we still have the category ${ }^{*}{ }_{-} \bmod _{\mathcal{D}}(\mathcal{A})$ of ${ }^{*}$-representations of $\mathcal{A}$ on inner product $\mathcal{D}$-modules.

We shall now show that a completely positive inner product $\langle\cdot, \cdot\rangle_{\mathcal{D}}$ on $\mathcal{H}_{\mathcal{D}}$ can indeed be identified with a certain completely positive map. To this end recall that the complex conjugate $\mathcal{D}_{\text {-module }}{ }_{\mathcal{D}} \overline{\mathcal{H}}$ is defined to be $\overline{\mathcal{H}}=\mathcal{H}$ as R-module but now the $\mathrm{C}$ - and $\mathcal{D}$-module structure are defined by

$$
\alpha \bar{x}=\overline{x \bar{\alpha}} \text { and } \quad d \cdot \bar{x}=\overline{x \cdot d^{*}},
$$

where $x \mapsto \bar{x}$ denotes the identity map $\mathcal{H} \longrightarrow \overline{\mathcal{H}}$. This way, ${ }_{\mathcal{D}} \overline{\mathcal{H}}$ becomes a left $\mathcal{D}$-module. Then we consider the following $(\mathcal{D}, \mathcal{D})$-bimodule ${ }_{\mathcal{D}} \mathcal{E}_{\mathcal{D}}={ }_{\mathcal{D}} \overline{\mathcal{H}} \otimes \otimes_{\mathcal{C}} \mathcal{H}_{\mathcal{D}}$. The map $I:_{\mathcal{D}} \mathcal{E}_{\mathcal{D}} \longrightarrow{ }_{\mathcal{D}} \mathcal{E}_{\mathcal{D}}$ defined by

$$
I: \bar{x} \otimes y \mapsto \bar{y} \otimes x
$$

is a $\mathrm{C}$-antilinear $(\mathcal{D}, \mathcal{D})$-antibimodule involution, i.e. we have $I\left(d \cdot z \cdot d^{\prime}\right)=d^{\prime *} \cdot I(z) \cdot d^{*}$ for all $d, d^{\prime} \in \mathcal{D}$ and $z \in{ }_{\mathcal{D}} \mathcal{E}_{\mathcal{D}}$. Moreover, $\mathcal{H}^{n}$ becomes canonically a right $M_{n}(\mathcal{D})$-module whence $M_{n}(\mathcal{E})=\overline{\mathcal{H}^{n}} \otimes_{\mathrm{C}} \mathcal{H}^{n}$ is a $\left(M_{n}(\mathcal{D}), M_{n}(\mathcal{D})\right)$-bimodule in a canonical way. Clearly $I$ extends to $M_{n}(\mathcal{E})$ by $I(z)_{i j}=I\left(z_{j i}\right)$ for a matrix $z=\left(z_{i j}\right) \in M_{n}(\mathcal{E})$. Still, we have $I\left(D \cdot z \cdot D^{\prime}\right)=\left(D^{\prime}\right)^{*} \cdot I(z) \cdot D^{*}$ for $D, D^{\prime} \in M_{n}(\mathcal{D})$ and $z \in M_{n}(\mathcal{E})$. Now consider the subset $\mathcal{K}_{n}(\mathcal{E})$ consisting of convex combinations of elements $z \in M_{n}(\mathcal{E})$ with $z_{i j}=\overline{x_{i}} \otimes x_{j}$ where $x_{1}, \ldots, x_{n} \in \mathcal{H}$. Clearly $I\left(\mathcal{K}_{n}(\mathcal{E})\right)=\mathcal{K}_{n}(\mathcal{E})$ and $D^{*} \mathcal{K}_{n}(\mathcal{E}) D \subseteq \mathcal{K}_{n}(\mathcal{E})$ for all $D \in M_{n}(\mathcal{D})$. This way, $\mathcal{E}$ becomes a matrix-ordered space in the sense of $[35$, Def. 11.1.1], even in the sense of $(\mathcal{D}, \mathcal{D})$-bimodules, i.e. $C$ replaced by $\mathcal{D}$ everywhere. This allows to speak of a completely positive map $\Phi: \mathcal{E} \longrightarrow \mathcal{D}$, i.e. a $(\mathcal{D}, \mathcal{D})$-bimodule map such that $\Phi(z)^{*}=\Phi(I(z))$ and

$$
\Phi\left(\mathcal{K}_{n}(\mathcal{E})\right) \subseteq M_{n}(\mathcal{D})^{+}
$$

for all $n \in \mathbb{N}$. Again, we have to extend the definition of completely positive maps as in [35, Def. 11.1.3] to the case of $(\mathcal{D}, \mathcal{D})$-bimodules.

Proposition 4.1 Let $\mathcal{H}_{\mathcal{D}}$ be a right $\mathcal{D}$-module. Then a $\mathcal{D}$-valued inner product is equivalent to a $(\mathcal{D}, \mathcal{D})$-bimodule map $\Phi:{ }_{\mathcal{D}} \mathcal{E}_{\mathcal{D}}={ }_{\mathcal{D}} \overline{\mathcal{H}} \otimes_{\mathcal{C}} \mathcal{H}_{\mathcal{D}} \longrightarrow \mathcal{D}$ with $\Phi(z)^{*}=\Phi(I(z))$ via

$$
\langle x, y\rangle_{\mathcal{D}}=\Phi(\bar{x} \otimes y) .
$$

Moreover, $\langle\cdot, \cdot\rangle_{\mathcal{D}}$ is completely positive iff $\Phi$ is completely positive.

Proof: This is now a straightforward verification.

We turn now to tensor products. Consider ${ }_{\mathcal{B}} \mathcal{E}_{\mathcal{A}} \in{ }^{*}{ }^{-} \bmod _{\mathcal{A}}(\mathcal{B})$ and ${ }_{\mathrm{e}} \mathcal{F}_{\mathcal{B}} \in{ }^{*}{ }_{-} \bmod _{\mathcal{B}}(\mathcal{C})$. Then on ${ }_{e} \mathcal{F}_{\mathcal{B}} \otimes_{\mathcal{B}}{ }_{B} \mathcal{E}_{\mathcal{A}}$, which is a $(\mathcal{C}, \mathcal{A})$-bimodule, one has a $\mathcal{A}$-valued inner product defined by

$$
\left\langle x_{1} \otimes y_{1}, x_{2} \otimes y_{2}\right\rangle_{\mathcal{A}}^{\mathcal{F} \otimes \mathcal{E}}=\left\langle y_{1},\left\langle x_{1}, x_{2}\right\rangle_{\mathcal{B}}^{\mathcal{F}} \cdot y_{2}\right\rangle_{\mathcal{A}}^{\varepsilon} \text {. }
$$

As $\langle\cdot, \cdot\rangle_{\mathcal{A}}^{\mathcal{F} \otimes \mathcal{E}}$ may be degenerate one needs to divide by the degeneracy space which is possible as it is invariant under the $(\mathcal{C}, \mathcal{A})$-bimodule structure. As quotient one obtains an inner product $\mathcal{A}$-module $e_{e} \mathcal{F}_{\mathcal{B}} \widehat{\otimes}_{\mathcal{B}}{ }_{\mathcal{B}} \mathcal{E}_{\mathcal{A}}$ with a ${ }^{*}$-representation of $\mathcal{C}$. Hence one ends up with a functor

$$
\widehat{\otimes}_{\mathcal{B}}:{ }^{*}-\bmod _{\mathcal{B}}(\mathcal{C}) \times{ }^{*}-\bmod _{\mathcal{A}}(\mathcal{B}) \longrightarrow{ }^{*}-\bmod _{\mathcal{A}}(\mathcal{C}) .
$$


Clearly, $\widehat{\otimes}$ is associative up to the usual canonical isomorphisms. It turns out that completely positive inner products behave well under this tensor product $\left[17\right.$, Thm. 4.7] whence $\widehat{\otimes}_{\mathcal{B}}$ restricts to a functor

$$
\widehat{\otimes}_{\mathcal{B}}:{ }^{*}{ }_{-} \operatorname{rep}_{\mathcal{B}}(\mathcal{C}) \times{ }^{*}-\operatorname{rep}_{\mathcal{A}}(\mathcal{B}) \longrightarrow{ }^{*}{ }_{-} \operatorname{rep}_{\mathcal{A}}(\mathcal{C}) .
$$

By fixing e.g. the first argument of $\widehat{\otimes}_{\mathcal{B}}$ one arrives at the algebraic analog of Rieffel's induction procedure for ${ }^{*}$-representations of $C^{*}$-algebras [33,34], see also [17, Ex. 4.9], which itself can be seen as a generalization [14, Prop. 4.7] of the well-known GNS construction of *-representations out of a positive linear functional, see e.g. any textbook on $C^{*}$-algebras and [35, Sect 8.6] for a detailed exposition in the context of ${ }^{*}$-algebras over $\mathbb{C}$ and [9] for applications to deformation quantization.

\section{Strong Morita equivalence and the Picard groupoid}

Morita equivalence was first developed by Morita [29] in the purely algebraic framework of associative rings, see e.g. [3]. Later, Rieffel transfered these ideas to $C^{*} / W^{*}$-algebras leading to the notion of strong Morita equivalence [33,34]. Ara specialised the general notion to rings with involution coining the notion of *-Morita equivalence [1,2] and Bursztyn-Waldmann discussed the case of *algebras over $C=R(i)$, generalizing Rieffel's strong Morita equivalence to this situation, therefor also called strong Morita equivalence [14,17]. A reasonable notion of Morita equivalence in the last class of algebras in the list in the introduction, the ${ }^{*}$-algebras over $\mathbb{C}$, seems still missing. Here one wants to go beyond the purely algebraic treatment taking into account the much more refined notions of ${ }^{*}$-representation theory available here [35]. In particular, given some locally convex topologies on the ${ }^{*}$-algebras, one would like to have some notion of Morita equivalence respecting these extra structures.

For $C^{*}$-algebras all the possible notions of Morita equivalence give the same equivalence relation, see $[2,5,13]$ while usually one only has the implications

$$
\text { Strong Morita equivalence } \Rightarrow{ }^{*} \text {-Morita equivalence } \Rightarrow \text { Morita equivalence. }
$$

Star product algebras are shown to behave like $C^{*}$-algebras concerning (5.1): here also the ringtheoretical Morita equivalence implies strong Morita equivalence [16]. However, the situation becomes more complicated if one asks in how many (essentially different) ways two ${ }^{*}$-algebras can be (*- resp. strongly) Morita equivalent. This information is encoded in the (*- resp. strong) Picard groupoid which we shall now describe.

Thus we first have to recall the definition of strong and *-Morita equivalence in some more detail. Let ${ }_{\mathcal{B}} \mathcal{E}_{\mathcal{A}}$ be a $(\mathcal{B}, \mathcal{A})$-bimodule endowed with a $\mathcal{A}$-valued inner product $\langle\cdot, \cdot\rangle_{\mathcal{A}}$ and a $\mathcal{B}$-valued inner product ${ }_{\mathcal{B}}\langle\cdot, \cdot\rangle$. As $\mathcal{B}$ acts on $\mathcal{E}$ from the left the $\mathcal{B}$-valued inner product is linear (to the left) in the first argument. An inner product $\langle\cdot, \cdot\rangle_{\mathcal{A}}$ is called $f u l l$ if the span of all $\langle x, y\rangle_{\mathcal{A}}$ in $\mathcal{A}$ coincides with $\mathcal{A}$. This is equivalent to say that the map $\Phi$ associated to $\langle\cdot, \cdot\rangle_{\mathcal{A}}$ as in Proposition 4.1 is surjective. The $(\mathcal{B}, \mathcal{A})$-bimodule ${ }_{\mathcal{B}} \mathcal{E}_{\mathcal{A}}$ with inner products ${ }_{\mathcal{B}}\langle\cdot, \cdot\rangle$ and $\langle\cdot, \cdot\rangle_{\mathcal{A}}$ is called a ${ }^{*}$-equivalence bimodule if $\mathcal{B} \cdot \mathcal{E}=\mathcal{E}=\mathcal{E} \cdot \mathcal{A}$, both inner products are non-degenerate and full, and they satisfy

$$
\langle x, b \cdot y\rangle_{\mathcal{A}}=\left\langle b^{*} \cdot x, y\right\rangle_{\mathcal{A}}, \quad{ }_{\mathcal{B}}\langle x \cdot a, y\rangle={ }_{\mathcal{B}}\left\langle x, y \cdot a^{*}\right\rangle, \quad \text { and } \quad{ }_{\mathcal{B}}\langle x, y\rangle \cdot z=x \cdot\langle y, z\rangle_{\mathcal{A}}
$$

for all $x, y, z \in \mathcal{E}, b \in \mathcal{B}$ and $a \in \mathcal{A}$. If in addition the inner products are completely positive then ${ }_{\mathcal{B}} \mathcal{E}_{\mathcal{A}}$ is called a strong equivalence bimodule, see $[1,17]$. If such a bimodule exists the algebras are called *-Morita equivalent or strongly Morita equivalent, respectively. It turns out that the $\widehat{\otimes}$-tensor product of equivalence bimodules is again an equivalence bimodule. This leads to the statements that *-Morita equivalence as well as strong Morita equivalence is indeed an equivalence relation within the class of *-algebras which are non-degenerate and idempotent. The condition of non-degeneracy and idempotency has to be put since otherwise both notions fail to be reflexive. In particular, unital *-algebras are always non-degenerate and idempotent. 
The strong Picard groupoid is now constructed as follows. $\operatorname{Pic}^{\operatorname{str}}(\mathcal{B}, \mathcal{A})$ consists of (isometric) isomorphism classes of equivalence bimodules ${ }_{\mathcal{B}} \mathcal{E}_{\mathcal{A}}$ and $\operatorname{Pic}^{\mathrm{str}}(\mathcal{B}, \mathcal{A})$ is viewed as space of arrows of a (large) groupoid with class of units given by the ${ }^{*}$-algebras themselves. The composition law

$$
\operatorname{Pic}^{\operatorname{str}}(\mathcal{C}, \mathcal{B}) \times \operatorname{Pic}^{\operatorname{str}}(\mathcal{B}, \mathcal{A}) \longrightarrow \operatorname{Pic}^{\text {str }}(\mathcal{C}, \mathcal{A})
$$

is given by the tensor product $\widehat{\otimes}$ and the units are the trivial bimodules ${ }_{\mathcal{A}} \mathcal{A}_{\mathcal{A}}$ with the canonical inner products $\langle a, b\rangle_{\mathcal{A}}=a^{*} b$ and ${ }_{\mathcal{A}}\langle a, b\rangle=a b^{*}$. Analogously one defines the ${ }^{*}$-Picard groupoid $\operatorname{Pic}^{*}(\cdot, \cdot)$ based on ${ }^{*}$-equivalence bimodules. It turns out that this defines indeed a groupoid $\operatorname{Pic}^{\text {str }}(\cdot, \cdot)$, see $[17$, Thm. 6.1] for an alternative construction and e.g. [3,6] for the ring-theoretical Picard groupoid. Finally, one observes that Rieffel's original version of strong Morita equivalence for $C^{*}$-algebras (which involves additional completeness properties) gives a $C^{*}$-algebraic strong $\mathrm{Pi}$ card groupoid $\mathrm{Pic}_{C^{*}}^{\text {str }}$, see [10], which turns out to be equivalent to the considerably easier strong Picard groupoid $\mathrm{Pic}^{\text {str }}$, restricted to the corresponding Pedersen ideals, see [17, Cor. 6.11]. Thus $\mathrm{Pic}^{\text {str }}$ encodes the complete strong Morita theory for $C^{*}$-algebras.

In general, the understanding of Mortia theory is equivalent to the understanding of the Picard groupoid in each of its flavours above. There are two basic questions:

i.) Which *-algebras $\mathcal{A}, \mathcal{B}$ are strongly Morita equivalent, i.e. for which is $\operatorname{Pic}^{\operatorname{str}}(\mathcal{B}, \mathcal{A}) \neq \emptyset$ ?

ii.) How many different self-equivalences does $\mathcal{A}$ have, i.e. what is the strong Picard group $\operatorname{Pic}^{\operatorname{str}}(\mathcal{A})=\operatorname{Pic}^{\text {str }}(\mathcal{A}, \mathcal{A})$ of $\mathcal{A}$ ?

Konwing this we have a complete description of $\operatorname{Pic}^{\text {str }}(\cdot, \cdot)$ as it is a groupoid and a groupoid is determined by its orbits and isotropy groups. In particular, if $\operatorname{Pic}^{\operatorname{str}}(\mathcal{B}, \mathcal{A}) \neq \emptyset$ then $\operatorname{Pic}^{\operatorname{str}}(\mathcal{B}, \mathcal{A}) \cong$ $\operatorname{Pic}^{\text {str }}(\mathcal{A})$ as sets since isotropy groups (here $\operatorname{Pic}^{\text {str }}(\mathcal{A})$ ) act transitively and freely on the spaces of arrows. Hence in this situation $\operatorname{Pic}^{\operatorname{str}}(\mathcal{A}) \cong \operatorname{Pic}^{\text {str }}(\mathcal{B})$ as groups where each element of $\operatorname{Pic}^{\text {str }}(\mathcal{B}, \mathcal{A})$ provides an isomorphism.

Another type of question is how the different flavours of Picard groupoids for ${ }^{*}$-algebras over C are related. It is rather easy to see that 'forgetting' the positivity of the inner products or 'forgetting' the inner products at all yields groupoid morphisms

$$
\mathrm{Pic}^{\text {str }} \longrightarrow \mathrm{Pic}^{*}, \quad \mathrm{Pic}^{\text {str }} \longrightarrow \mathrm{Pic}, \quad \text { and } \mathrm{Pic}^{*} \longrightarrow \mathrm{Pic}
$$

which are in general neither injective nor surjective. The morphism $\mathrm{Pic}^{\text {str }} \longrightarrow \mathrm{Pic}^{*}$ usually fails to be surjective as there may be also other inner products on equivalence bimodules having different 'signatures'. The morphism Pic* $\longrightarrow$ Pic fails to be injective for the same reason. However, also $\mathrm{Pic}^{\text {str }} \longrightarrow$ Pic shows a rather rich and complicated behaviour, even for very 'nice' classes of ${ }^{*}$-algebras like $C^{*}$-algebras. For $C^{*}$-algebras as well as for star product algebras one can prove injectivity but surjectivity fails in general. The defect of being surjective can be described rather explicit in terms of certain non-inner automorphisms, see [17, Sect. 7,8$]$ for a detailed analysis and examples.

The last type of question we want to mention is about formal deformations of *algebras. Thus assume that $\mathcal{A}=(\mathcal{A}[[\lambda]], \star)$ and $\mathcal{B}=\left(\mathcal{B}[[\lambda]], \star^{\prime}\right)$ are Hermitian formal deformations of $\mathcal{A}, \mathcal{B}$. Then one wants to relate $\operatorname{Pic}^{\operatorname{str}}(\mathcal{B}, \mathcal{A})$ and $\operatorname{Pic}^{\mathrm{str}}(\mathcal{B}, \mathcal{A})$. Here one heavily uses the fact that formal deformations and ordered rings fits together nicely as $\mathrm{R}[[\lambda]]$ is canonically ordered again. While for $\operatorname{Pic}(\cdot, \cdot)$ and $\operatorname{Pic}^{*}(\cdot, \cdot)$ it is rather easy to see that the 'classical limit' map cl, which sets $\lambda=0$, induces a groupoid morphism $\mathrm{cl}_{*}$. This turns out to be more complicated in the case of $\operatorname{Pic}^{\operatorname{str}}(\cdot, \cdot)$. Here it is only true for completely positive deformations. Recall that a Hermitian deformation $\mathcal{A}$ is called completely positive if for every C-linear positive functional $\Omega_{0}: M_{n}(\mathcal{A}) \longrightarrow \mathrm{C}$ one finds a deformation into a $\mathrm{C}[[\lambda]]$-linear positive functional $\Omega=\Omega_{0}+o(\lambda): M_{n}(\mathcal{A}) \longrightarrow \mathrm{C}[[\lambda]]$. Luckily, 
Hermitian star products are always completely positive deformations [17, Thm. 8.5] whence we have a groupoid morphism

$$
\mathrm{cl}_{*}: \operatorname{Pic}^{\mathrm{str}}(\mathcal{B}, \mathcal{A}) \longrightarrow \operatorname{Pic}^{\text {str }}(\mathcal{B}, \mathcal{A})
$$

for star product algebras and, more generally, completely positive deformations. This actually follows from the considerations in [14, Lem. 9.3]. Again, for all three versions Pic, Pic* and Pic ${ }^{\text {str }}$ the classical limit $\mathrm{cl}_{*}$ shows a rich and non-trivial behaviour, see [15] for a detailed discussion of the case of Pic and $[16,25]$ for a classification of (strongly) Morita equivalent star products resulting from these considerations concerning the classical limit map.

\section{The Picard groupoid in action}

In this last section we illustrate how one can obtain Morita invariants of algebras by looking at 'actions' of the Picard groupoids. We shall not give an axiomatic theory for actions of a large groupoid (or even bigroupoid) but mention just three examples, the results of which are classical:

i.) As already mentioned $\operatorname{Pic}^{\operatorname{str}}(\mathcal{A})$ is an invariant under strong Morita equivalence. This can be seen as result of the groupoid action of $\mathrm{Pic}^{\text {str }}$

$$
\operatorname{Pic}^{\text {str }}(\mathcal{B}, \mathcal{A}) \times \operatorname{Pic}^{\text {str }}(\mathcal{A}) \longrightarrow \operatorname{Pic}^{\text {str }}(\mathcal{B})
$$

on its isotropy groups by simply using the left multiplications.

ii.) Let $\mathcal{A}, \mathcal{B}$ be unital. Then the Hermitian $K_{0}$-group of $\mathcal{A}$ is the Grothendieck group $K_{0}^{H}(\mathcal{A})$ of the semi-group of isometric isomorphism classes of finitely generated projective right $\mathcal{A}$ modules with strongly non-degenerate completely positive inner products. Here strongly non-degenerate means that $x \in \mathcal{E} \mapsto\langle x, \cdot\rangle_{\mathcal{A}} \in \mathcal{E}^{*}=\operatorname{Hom}_{\mathcal{A}}(\mathcal{E}, \mathcal{A})$ is a bijection. Now let ${ }_{\mathcal{B}} \mathcal{E}_{\mathcal{A}}$ be a strong equivalence bimodule and let $\mathcal{H}_{\mathcal{B}}$ be a finitely generated projective preHilbert module with strongly non-degenerate inner product. Then $\mathcal{H}_{\mathcal{A}}^{\prime}=\mathcal{H}_{\mathcal{B}} \widehat{\otimes}_{\mathcal{B}}{ }_{\mathcal{B}} \mathcal{E}_{\mathcal{A}}$ is again finitely generated and projective as right $\mathcal{A}$-module and the completely positive inner product is again strongly non-degenerate. Passing to isometric isomorphism classes this yields a 'groupoid action'

$$
K_{0}^{H}(\mathcal{B}) \times \operatorname{Pic}^{\operatorname{str}}(\mathcal{B}, \mathcal{A}) \longrightarrow K_{0}^{H}(\mathcal{A})
$$

of the strong Picard groupoid on the Hermitian $K_{0}$-groups of unital ${ }^{*}$-algebras over $C$. From this we can see two things: first $K_{0}(\mathcal{A})$ carries a natural $\operatorname{Pic}^{\operatorname{str}}(\mathcal{A})$-representation, as the tensor product $\widehat{\otimes}_{\mathcal{A}}$ is clearly compatible with the direct orthogonal sum, and second $K_{0}^{H}(\mathcal{A}) \cong$ $K_{0}^{H}(\mathcal{B})$ as $\operatorname{Pic}^{\text {str }}(\mathcal{A}) \cong \operatorname{Pic}^{\operatorname{str}}(\mathcal{B})$ representation spaces. This observation was brought to our attention by Alan Weinstein.

iii.) Let $\mathcal{D}$ be an auxilliary ${ }^{*}$-algebra and denote by ${ }^{*}$ - $\operatorname{Rep}_{\mathcal{D}}(\mathcal{A})$ those ${ }^{*}$-representations $(\mathcal{H}, \pi) \in$ ${ }^{*}{ }_{-} \operatorname{rep}_{\mathcal{D}}(\mathcal{A})$ where $\pi(\mathcal{A}) \mathcal{H}=\mathcal{H}$. Then given a strong equivalence bimodule ${ }_{\mathcal{B}} \mathcal{E}_{\mathcal{A}}$ and $(\mathcal{H}, \pi) \in$ ${ }^{*} \operatorname{-Rep}_{\mathcal{D}}(\mathcal{A})$ we obtain ${ }_{\mathcal{B}} \mathcal{E}_{\mathcal{A}} \widehat{\otimes}_{\mathcal{A}} \mathcal{H} \in{ }^{*}{ }_{-} \operatorname{Rep}_{\mathcal{D}}(\mathcal{B})$ and the fucntor $\mathcal{H} \mapsto{ }_{\mathcal{B}} \mathcal{E}_{\mathcal{A}} \widehat{\otimes}_{\mathcal{A}} \mathcal{H}$ implements an equivalence of the categories ${ }^{*}-\operatorname{Rep}_{\mathcal{D}}(\mathcal{A})$ and ${ }^{*}{ }_{-}-\operatorname{Rep}{ }_{\mathcal{D}}(\mathcal{B})$, see $[17$, Cor. 5.16]. Note that this was one of the original motivations for the definition of strong Morita equivalence. Reinterpreting this Morita invariant we see that this comes from an 'action'

$$
\operatorname{Pic}^{\operatorname{str}}(\mathcal{B}, \mathcal{A}) \times{ }^{*}-\operatorname{Rep}_{\mathcal{D}}(\mathcal{A}) \longrightarrow{ }^{*}-\operatorname{Rep}_{\mathcal{D}}(\mathcal{B})
$$

which, however, is only defined up to natural isomorphisms and unitary equivalences. Thus the probably better framework would be to consider the Picard bigroupoid instead, i.e not identifying bimodules up to isomorphism, see [6]. Then (6.3) would better read as an 'action' of the Picard bigroupoid on the categories of ${ }^{*}$-representations. 
Let us finally mention that the above examples have their well-known counterparts for Pic ${ }^{\text {str }}$ replaced by Pic or Pic* leading the known Morita invariants in these contexts. Beside the above examples there are other Morita invariants which can be obtained by actions of the Picard groupoid like the moduli spaces of formal (Hermitian) deformations $\operatorname{Def}(\mathcal{A})$, see the discussion in [15, Sect. 3.3] and [11].

\section{References}

[1] ARA, P.: Morita equivalence for rings with involution. Alg. Rep. Theo. 2 (1999), 227-247.

[2] ArA, P.: Morita equivalence and Pedersen Ideals. Proc. AMS 129.4 (2000), 1041-1049.

[3] Bass, H.: Algebraic K-theory. W. A. Benjamin, Inc., New York, Amsterdam, 1968.

[4] Bayen, F., Flato, M., Frønsdal, C., Lichnerowicz, A., Sternheimer, D.: Deformation Theory and Quantization. Ann. Phys. 111 (1978), 61-151.

[5] BeER, W.: On Morita equivalence of nuclear $C^{*}$-algebras. J. Pure Appl. Algebra 26.3 (1982), $249-267$.

[6] BÉnabou, J.: Introduction to Bicategories. In: Reports of the Midwest Category Seminar, 1-77. Springer-Verlag, 1967.

[7] Bertelson, M., Cahen, M., Gutt, S.: Equivalence of Star Products. Class. Quant. Grav. 14 (1997), A93A107.

[8] Bordemann, M., Neumaier, N., Pflaum, M. J., Waldmann, S.: On representations of star product algebras over cotangent spaces on Hermitian line bundles. J. Funct. Anal. 199 (2003), 1-47.

[9] Bordemann, M., Waldmann, S.: Formal GNS Construction and States in Deformation Quantization. Commun. Math. Phys. 195 (1998), 549-583.

[10] Brown, L. G., Green, P., Rieffel, M.: Stable Isomorphism and Strong Morita Equivalence of $C^{*}-$ Algebras. Pacific J. Math. 71 (1977), 349-363.

[11] Bursztyn, H.: Semiclassical geometry of quantum line bundles and Morita equivalence of star products. Int. Math. Res. Not. 2002.16 (2002), 821-846.

[12] Bursztyn, H., Radko, O.: Gauge Equivalence of Dirac Structures and Symplectic Groupoids. Ann. Inst. Fourier 53 (2003), 309-337.

[13] Bursztyn, H., Waldmann, S.: ${ }^{*}$-Ideals and Formal Morita Equivalence of ${ }^{*}$-Algebras. Int. J. Math. 12.5 (2001), 555-577.

[14] Bursztyn, H., Waldmann, S.: Algebraic Rieffel Induction, Formal Morita Equivalence and Applications to Deformation Quantization. J. Geom. Phys. 37 (2001), 307-364.

[15] Bursztyn, H., Waldmann, S.: Bimodule deformations, Picard groups and contravariant connections. Preprint (Freiburg FR-THEP 2002/10) math.QA/0207255 (July 2002), 32 pages. To appear in K-Theory.

[16] Bursztyn, H., Waldmann, S.: The characteristic classes of Morita equivalent star products on symplectic manifolds. Commun. Math. Phys. 228 (2002), 103-121.

[17] Bursztyn, H., Waldmann, S.: Completely positive inner products and strong Morita equivalence. Preprint (FR-THEP 2003/12) math.QA/0309402 (September 2003), 36 pages.

[18] Bursztyn, H., Weinstein, A.: Picard groups in Poisson geometry. Preprint math.SG/0304048 (2003), 25 pages. To appear in Moscow Math. J.

[19] Cattaneo, A., Felder, G.: A Path Integral Approach to the Kontsevich Quantization Formula. Commun. Math. Phys. 212 (2000), 591-611.

[20] DeWilde, M., Lecomte, P. B. A.: Existence of Star-Products and of Formal Deformations of the Poisson Lie Algebra of Arbitrary Symplectic Manifolds. Lett. Math. Phys. 7 (1983), 487-496.

[21] Dito, G., Sternheimer, D.: Deformation quantization: genesis, developments and metamorphoses. In: Halbout, G. (EDS.): Deformation quantization, vol. 1 in IRMA Lectures in Mathematics and Theoretical Physics, 9-54. Walter de Gruyter, Berlin, New York, 2002.

[22] Fedosov, B. V.: A Simple Geometrical Construction of Deformation Quantization. J. Diff. Geom. 40 (1994), $213-238$.

[23] Gutt, S.: Variations on deformation quantization. In: Dito, G., Sternheimer, D. (EDs.): Conférence Moshé Flato 1999. Quantization, Deformations, and Symmetries, Mathematical Physics Studies no. 21, 217254. Kluwer Academic Publishers, Dordrecht, Boston, London, 2000. 
[24] HAag, R.: Local Quantum Physics. Springer-Verlag, Berlin, Heidelberg, New York, 2. edition, 1993.

[25] JurČO, B., Schupp, P., Wess, J.: Noncommutative Line Bundles and Morita Equivalence. Lett. Math. Phys. 61 (2002), 171-186.

[26] Kontsevich, M.: Deformation Quantization of Poisson Manifolds, I. Preprint q-alg/9709040 (September 1997).

[27] Lance, E. C.: Hilbert $C^{*}$-modules. A toolkit for operator algebraists, vol. 210 in London Mathematical Society Lecture Note Series. Cambridge University Press, Cambridge, 1995.

[28] Landsman, N. P.: Mathematical Topics between Classical and Quantum Mechanics. Springer Monographs in Mathematics. Springer-Verlag, Berlin, Heidelberg, New York, 1998.

[29] MoRItA, K.: Duality for modules and its applications to the theory of rings with minimum condition. Sci. Rep. Tokyo Kyoiku Daigaku Sect. A 6 (1958), 83-142.

[30] Nest, R., Tsygan, B.: Algebraic Index Theorem. Commun. Math. Phys. 172 (1995), 223-262.

[31] Omori, H., Maeda, Y., Yoshioka, A.: Weyl Manifolds and Deformation Quantization. Adv. Math. 85 (1991), 224-255.

[32] Raeburn, I., Williams, D. P.: Morita equivalence and continuous-trace $C^{*}$-algebras, vol. 60 in Mathematical Surveys and Monographs. American Mathematical Society, Providence, RI, 1998.

[33] Rieffel, M. A.: Induced representations of $C^{*}$-algebras. Adv. Math. 13 (1974), 176-257.

[34] Rieffel, M. A.: Morita equivalence for $C^{*}$-algebras and $W^{*}$-algebras. J. Pure. Appl. Math. 5 (1974), 51-96.

[35] Schmüdgen, K.: Unbounded Operator Algebras and Representation Theory, vol. 37 in Operator Theory: Advances and Applications. Birkhäuser Verlag, Basel, Boston, Berlin, 1990.

[36] Weinstein, A., Xu, P.: Hochschild cohomology and characteristic classes for star-products. In: KHOVAnsKiJ, A., Varchenko, A., Vassiliev, V. (eds.): Geometry of differential equations. Dedicated to V. I. Arnold on the occasion of his 60th birthday, 177-194. American Mathematical Society, Providence, 1998.

[37] Xu, P.: Morita Equivalence of Poisson Manifolds. Commun. Math. Phys. 142 (1991), 493-509. 